\title{
Desenvolvendo um modelo sintético de baixo custo para treinamento de drenagem torácica em ambiente de simulação
}

\author{
Developing a low cost model for chest drainage simulate training
}

\author{
Maria Allyce de Oliveira ${ }^{1}$, Erika Feitosa Queiroz ${ }^{1}$, Daniel Araujo Kramer de Mesquita ${ }^{1}$, \\ Luiza Matias Marques ${ }^{1}$, Fernanda Martins Maia $^{2}$, Rafaela Vieira Correa ${ }^{3}$
}

Oliveira MA, Queiroz EF, Mesquita DAK, Marques LM, Maia FM, Correa RV. Desenvolvendo um modelo sintético de baixo custo
para treinamento de drenagem torácica em ambiente de simulação / Developing a low cost model for chest drainage simulate training. Rev Med (São Paulo). 2020 mar.-abr.;99(2):115-21.

RESUMO: Objetivo: Descrever a construção de um simulador de baixo custo de drenagem torácica para capacitar a realização deste procedimento. Métodos: A concepção do modelo se deu em 2014 por integrantes do Programa de Educação Tutorial do curso de Medicina da UNIFOR. A seguir, o modelo foi testado por 10 especialistas e posteriormente aprovado para ser aplicado em quatro edições de um curso teórico-prático de procedimentos invasivos com acadêmicos de Medicina de diferentes universidades. Para avaliar o simulador e o desempenho no seu uso, aplicou-se questionários semiestruturados e checklist. Os dados foram analisados pelo Statistical Package for the Social Sciences v. 22 usando estatística descritiva. Resultados: Usou-se um manequim plástico comercial em que foi realizado um corte de $8 \times 8 \mathrm{~cm}$ na região lateral do tórax, correspondente ao local da drenagem. Neste local, foram posicionadas folhas de E.V.A. (simulando pele e músculos intercostais), esponja de estofado (subcutâneo) e folha de PVC (pleura). Na parte interna do manequim foi colocada uma estrutura de madeira contendo duas costelas e, entre elas, um copo plástico recoberto por E.V.A. contendo líquido vermelho (simulando um hemotórax). O custo inicial do modelo completo foi de R \$ 81,00/US\$21,00. Quando testado por especialistas, todos concordaram que o modelo pode ser usado para o ensino na graduação. Durante o curso de procedimentos invasivos, 129 acadêmicos realizaram o procedimento, em que $64,3 \%$ dos alunos inseriram corretamente o dreno e $79,1 \%$ conectaram ao sistema de drenagem. Apesar de um percentual de erros importante visto em passos iniciais básicos, os alunos conseguiram executar a técnica proposta de forma suficiente. Conclusão: O simulador de drenagem de tórax mostrou-se ser de fácil acesso e reprodução nas universidades, $o$ que o torna uma ferramenta útil para o ensino.

Descritores: Educação médica; Simulação; Ensino; Drenagem; Tubos torácicos; Treinamento por simulação.

\begin{abstract}
Objective: To describe the construction of a lowcost simulator of chest drainage for the training of undergraduate medical students. Methods: The model was conceived in 2014 by members of the Tutorial Education Program - UNIFOR. It was tested by 10 specialists and approved for application in four editions of a theory and practice course on invasive procedures with medical students from different universities. To evaluate the simulator and its performance, semi-structured questionnaires and checklists were applied. Data were analyzed in the Statistical Package for the Social Sciences v. 22, using descriptive statistics. Results: A commercial mannequin was used to build the model. An $8 \times 8 \mathrm{~cm}$ square cut was made in the lateral wall of the chest. Inside this region, EVA sheets (simulating skin and intercostal muscles), foam padding (subcutaneous tissue) and transparent PVC sheet (pleura) were placed. In the inner part of the mannequin, a wooden structure containing two ribs was constructed and a plastic cup containing red-dyed water was placed between the two ribs. The complete model had an initial cost of $\mathrm{R} \$ 81,00 /$ US $\$ 21,00$. The medical specialists agreed $(100 \%)$ that it can be used for undergraduate teaching. During the course on invasive procedures, 129 students performed the procedure; $64.3 \%$ of them were able to correctly insert the drain and $79.1 \%$ connected it to the drainage system. Despite of the high percentage of errors in basic initial steps, the students were able to execute the technique satisfactorily. Conclusion: The low-cost chest drainage simulator was easy to access and to reproduce in universities, which makes it an important tool for teaching.
\end{abstract}

Keywords: Education, medical; Simulation technique; Teaching; Drainage; Chest tubes; Simulation training.

Realizdo na Universidade de Fortaleza (UNIFOR). Apresentado no XXXII Congresso Brasileiro de Cirurgia, São Paulo, SP, 28 abr. 01 maio, 2017.

1. Graduação no curso de Medicina, Universidade de Fortaleza, Fortaleza, CE, Brasil. Integrante do Programa de Educação Tutorial (PET) Medicina da UNIFOR. ORCID: Oliveira MA - https://orcid.org/0000-0001-7907-1132, Queiroz EF - https://orcid.org/0000-0001-8173-4820, Mesquita DAK https://orcid.org/0000-0002-6299-0826; Marques LM - https://orcid.org/0000-0002-8944-0496. Email: allycetx@gmail.com, erikafqueiroz@gmail. com, danielkramer@edu.unifor.br, luizamarques@edu.unifor.br

2. Neurologista, Docente do Curso de Medicina, Centro de Ciências da Saúde, Universidade de Fortaleza - UNIFOR, Fortaleza, CE, Brasil. ORCID: http://orcid.org/0000-0001-6548-7268. E-mail: fernandamaia@unifor.br.

3. Endocrinologista, Docente do Curso de Medicina e Tutora do Programa de Educação Tutorial (PET), Centro de Ciências da Saúde, Universidade de Fortaleza - UNIFOR, Fortaleza, CE, Brasil. ORCID: http://orcid.org/0000-0001-7840-2745. E-mail: rafaelavieiracorrea@gmail.com.

Endereço para correspondência: Maria Allyce de Oliveira. Rua Desembargador Floriano Benevides Magalhães, nº 221 - Bairro: Edson Queiroz.

Fortaleza, CE. CEP: 60811-905. E-mail: allycetx@gmail.com. 


\section{INTRODUÇÃO}

drenagem torácica é um procedimento médico de grande relevância no tratamento de intercorrências pleurais, sendo bastante utilizado tanto em situações eletivas, como em casos de urgência e emergência ${ }^{1,2}$.

Embora seja uma prática de, aparentemente, simples aplicabilidade nos diversos hospitais do país, os profissionais médicos devem estar aptos a realizar esse procedimento com técnica adequada ${ }^{2}$. Tal fato é corroborado pelas Diretrizes Curriculares Nacionais, que orientam que, no âmbito da clínica cirúrgica, o estudante deve aprender a técnica da drenagem de tórax e realizá-la, pelo menos, por meio de simulação ${ }^{3}$.

Sabe-se que o treinamento de procedimentos cirúrgicos proporciona a aquisição de habilidades específicas de forma segura e controlada por meio da simulação de situações reais. Portanto, busca-se construir modelos que possam se assemelhar à anatomia humana em seus detalhes, com baixo custo e fácil reprodutibilidade, para que se possa disseminar o ensino por meio da prática segura de procedimentos em unidades de saúde e em universidades ${ }^{4,5}$.

Há descrição na literatura da fabricação de modelos de baixo custo de drenagem torácica para o aprendizado prático em alternativa a modelos comerciais, porém estes utilizam animais ou outros materiais de difícil acesso para a obtenção dos simuladores ${ }^{6-9}$.

Em oposição a isso, este artigo apresenta um modelo mais barato do que as alternativas existentes para o treinamento de drenagem torácica, sem risco de contaminação com materiais biológicos. Assim, constitui um modelo que usa materiais sintéticos de baixo custo para simular o procedimento, apresentando um custo menor que R\$ 100,00, o que facilita o seu acesso e a sua reprodução em meio acadêmico.

Diante disso, o presente artigo busca descrever a construção de um manequim de drenagem de tórax com baixo custo e simples reprodução, assim como sua utilização para treinamento com foco na capacitação para a realização de tal procedimento.

\section{MATERIAL E MÉTODOS}

O primeiro passo deste estudo foi a concepção de modelos de drenagem torácica no ano de 2014 por alunos integrantes do Programa de Educação Tutorial (PET) Medicina da UNIFOR, utilizando como base um manequim plástico de baixo custo, semelhante aos usados em lojas comerciais. Após isso, foi realizado um planejamento de estimativa de custos e identificação de materiais acessíveis para simular a anatomia humana. Por fim, houve a construção do modelo propriamente dito com ajustes para simular a dinâmica do procedimento.

O próximo passo do estudo se deu com o teste do modelo por 10 médicos especialistas que atuam como preceptores na formação de residentes de cirurgia geral ou emergência, visando testar o manequim para o ensino em ambiente de simulação. Para avaliar o modelo, os médicos responderam um questionário semi-estruturado com aspectos quantitativos em escala Likert e, também, qualitativos.

Além disso, os autores elaboraram um checklist para avaliar o desempenho dos acadêmicos durante o treino, baseando-se em uma revisão de literatura que incluiu referências com informações detalhadas e didáticas sobre o passo a passo do procedimento ${ }^{2,10-12}$.

Após esse processo, o simulador foi usado durante quatro edições de um curso teórico-prático de procedimentos invasivos para alunos da graduação, idealizado pelo PET Medicina da UNIFOR, durante o período de 2015-2017 (Figura 1). O curso contou com aulas teóricas ministradas por tutores previamente treinados, contendo aspectos como: conceitos iniciais, indicações do procedimento e suas contraindicações, bem como o passo a passo da drenagem de tórax. Após isso, os alunos tiveram demonstração prática da técnica pelos tutores e, posteriormente, realizaram o procedimento individualmente, sendo avaliados por meio do checklist (Tabela 1).

Desse modo, a amostra do estudo incluiu também estudantes de Medicina de diferentes semestres, regularmente matriculados em instituições de ensino superior, que se inscreveram no curso teórico-prático e concordaram com o Termo de Consentimento Livre e Esclarecido.

Os dados coletados foram analisados por meio do Statistical Package for the Social Sciences (SPSS) v. 22 usando estatística descritiva. Este projeto foi aprovado pelo Comitê de Ética em Pesquisa da Universidade de Fortaleza sob o número CAAE: 30964514.0.0000.5052 e parecer número 688.827. Para avaliar a normalidade da amostra foi utilizado o teste Kolmogorov Smirnov, em que os valores com $\mathrm{p}<0.05$ foram considerados estatisticamente significativos. 


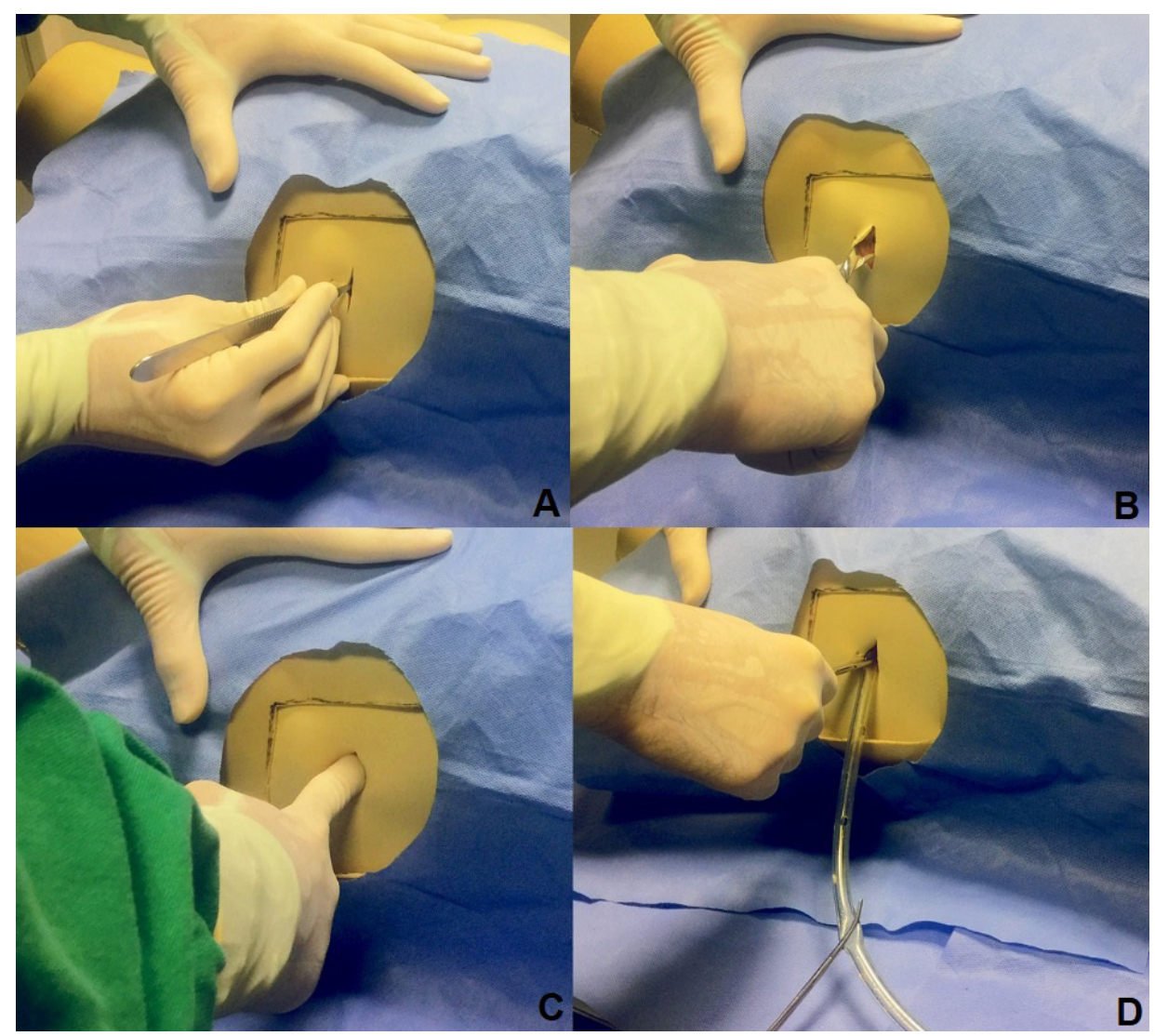

Figura 1. Modelo de drenagem de tórax evidenciando o passo-a-passo do procedimento. (A) Incisão da pele; (B) Divulsão dos músculos intercostais; (C) Exploração digital do orifício de drenagem; (D) Inserção do dreno. Fonte: Figura elaborada pelos autores

Tabela 1. Checklist de drenagem torácica

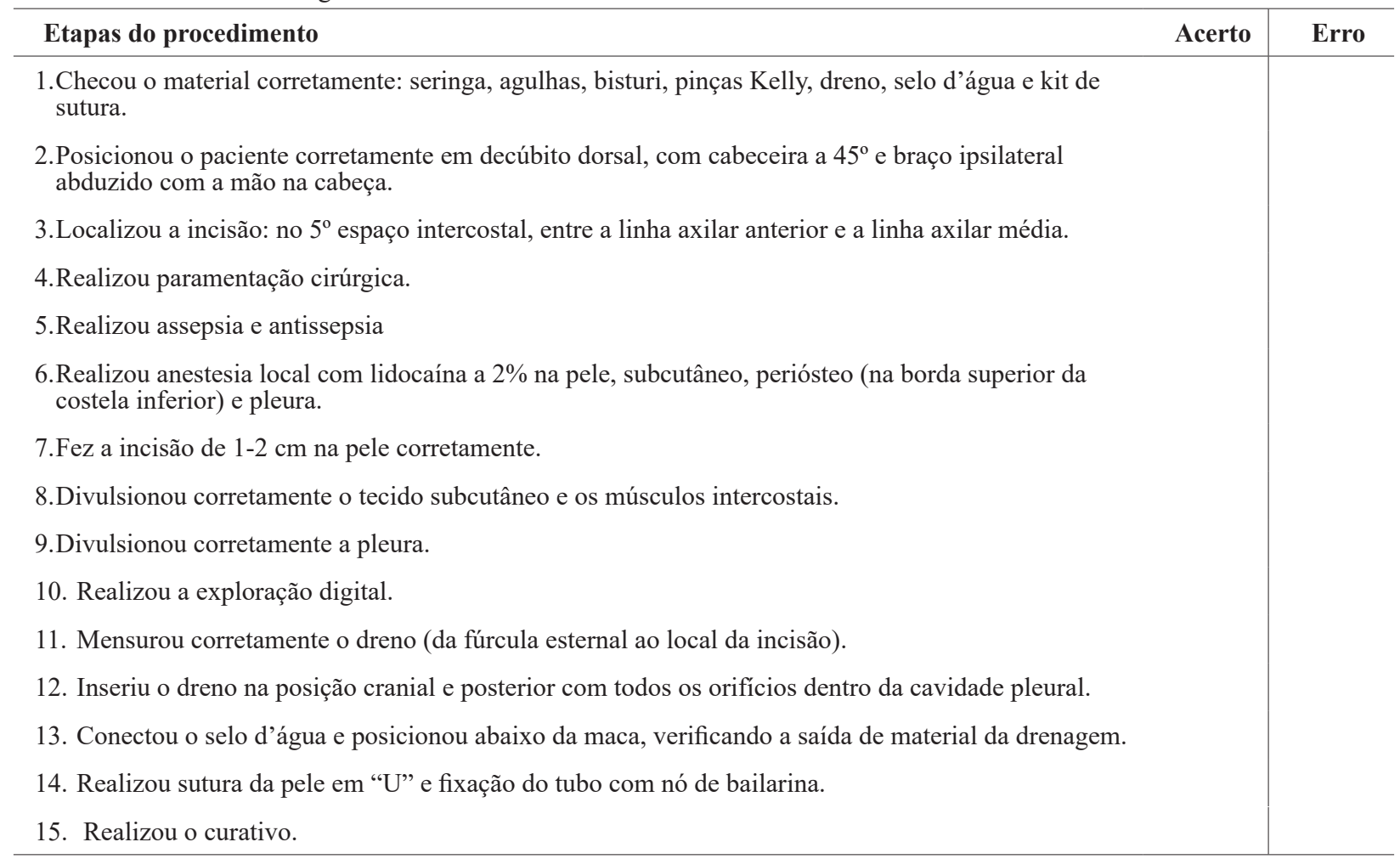




\section{RESULTADOS}

\section{Construção do modelo}

Foi realizado um corte quadrado de $8 \times 8 \mathrm{~cm}$ na parede lateral do tórax e, no interior desta região, foram adicionados blocos com camadas quadrangulares de $12 \times 12 \mathrm{~cm}$ que são descritas a seguir: a primeira camada, correspondente à pele, é feita com folha de E.V.A cor bege; para a segunda camada, que simula o tecido subcutâneo, usa-se esponja de estofado; já a terceira camada, simulando os músculos da parede torácica, é feita com duas folhas de E.V.A de cor vermelha; por fim, a última camada, representando a pleura, é produzida por meio de uma folha transparente de PVC.

Além disso, na parte interna do manequim foram colocadas duas madeiras retangulares, medindo cerca de 6 $\mathrm{cm}$, fixadas por meio de parafusos em uma base de madeira para simular duas costelas e o espaço intercostal onde deve ser inserido o dreno (Figura 2). Inicialmente, os autores do trabalho fabricaram o modelo para a prática da drenagem em uma situação clínica de hemotórax. Assim, nessa base de madeira foi acoplada um copo plástico, que se posiciona exatamente entre as duas costelas, recoberto por folha de E.V.A. na sua abertura superior. Esse copo contém água tingida de vermelho com tinta guache que funcionará como o conteúdo pleural hemorrágico. Dessa maneira, o dreno de tórax percorre as camadas que simulam os tecidos moles, passa por entre as duas costelas de madeira, adentra no dispositivo contendo água tingida de vermelho e com o auxílio de um sistema fechado a vácuo $\left(\right.$ Portovac $\left.^{\circledR}\right)$, o líquido desce pelo dreno.

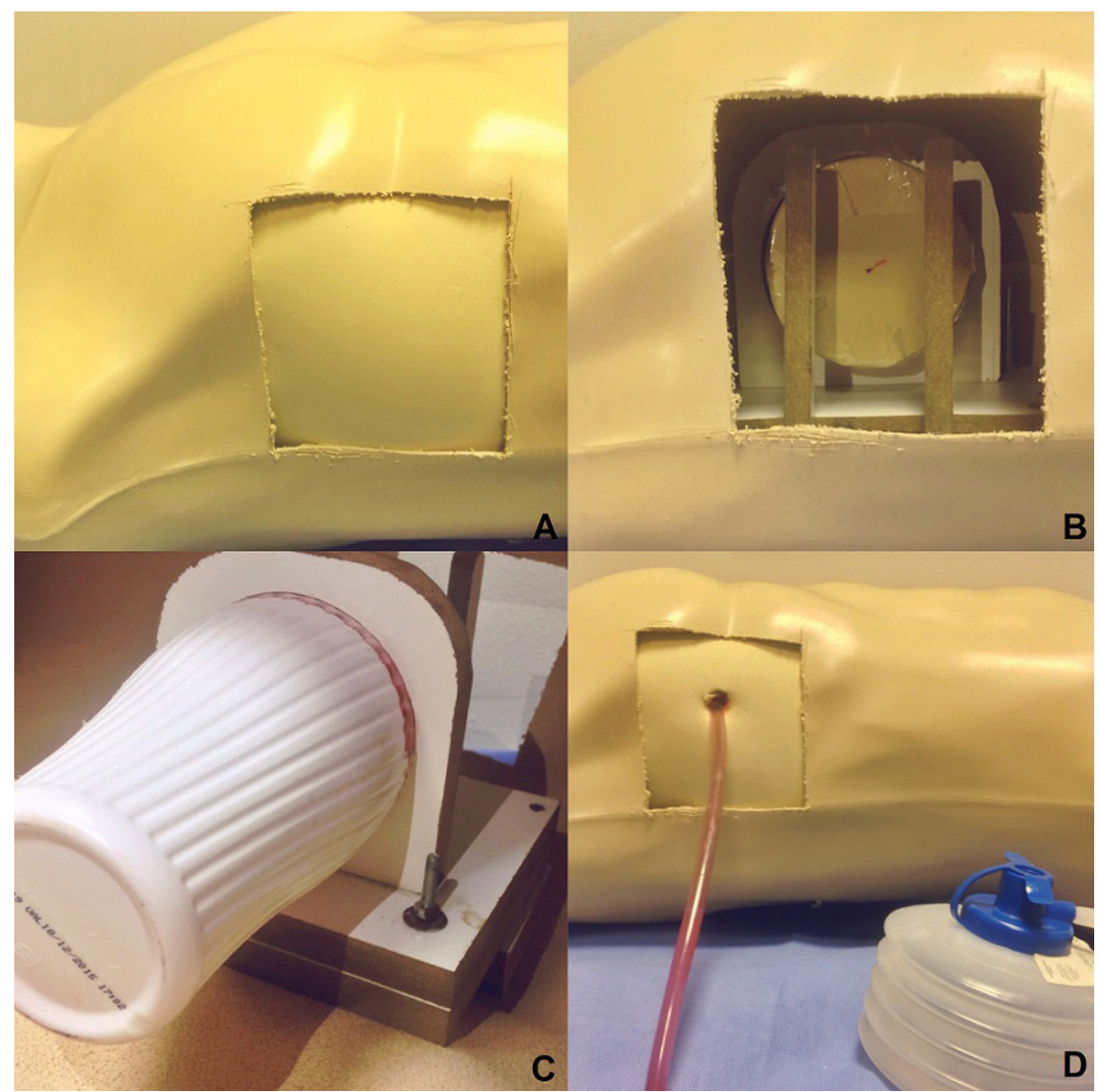

Fonte: Figura elaborada pelos autores.

Figura 2. Detalhes do simulador de drenagem torácica. (A) Modelo sintético e o local de drenagem; (B) Região interna do modelo evidenciando as costelas de madeira e o copo plástico que possui revestimento na abertura superior com um orifício para a entrada do dreno; (C) Copo fixado contendo líquido composto pela diluição de água e tinta guache vermelha; (D) Modelo demonstrando o dreno inserido, o sistema Portovac ${ }^{\circledR}$ e a drenagem do hemotórax

Foram utilizados materiais sintéticos de baixo custo, que estão especificados com os respectivos preços na Tabela 2, totalizando um valor de R \$ 81,00/US\$21,00 pelo simulador completo inicial. Foi visto que o simulador necessitou de uma manutenção antes de cada nova edição do curso para ajuste do suporte e das costelas de madeira (marcenaria) no valor de R \$ 15,00/US\$ 4,00, somando-se ainda um custo adicional de R \$,00/US\$ 1,00 para cada bloco que simula as partes moles da parede torácica (pele, subcutâneo, músculos e pleura). Em média, durante cada edição do curso de procedimentos cirúrgicos, são realizadas cerca de 50 drenagens torácicas. Assim, o gasto total para 
50 procedimentos, portanto, a cada edição do curso foi de $\mathrm{R} \$ 215,00 / \mathrm{US} \$ 55,00$. Até o momento do presente estudo, o modelo confeccionado teve a durabilidade de 4 edições do curso, totalizando cerca de 200 procedimentos.

Tabela 2. Materiais para confecção do manequim.

\begin{tabular}{lll}
\hline Estrutura Simulada & Material & Custo (R\$) \\
\hline Tórax & Manequim comercial de plástico & R \$ 30,00/US\$ 7,70 \\
Pele + Subcutâneo + Músculos & 3 E.V.A $(12 \times 12 \mathrm{~cm})+$ Esponja de estofado $(12 \times 12 \mathrm{~cm})$ & $\mathrm{R} \$ 3,50 / \mathrm{US} \$ 0,90$ \\
Pleura & Folha de PVC transparente $(12 \times 12 \mathrm{~cm})$ & $\mathrm{R} \$ 0,50 / \mathrm{US} \$ 0,12$ \\
Líquido da cavidade torácica & $\begin{array}{l}\text { Copo plástico com água }+ \text { suporte de madeira para } \\
\text { sustentação }+6 \text { parafusos }\end{array}$ & $\mathrm{R} \$ 47,00 / \mathrm{US} \$ 12,00$ \\
\hline
\end{tabular}

Legenda: *Custo de manutenção por curso semestral (50 procedimentos); R\$215,00/US\$ 55,00.

Teste e aplicação do simulador

O modelo foi testado por professores vinculados a universidades e programas de residência em Fortaleza, especializados em Cirurgia Geral (3), Cirurgia Digestiva
(3), Cirurgia Plástica (2) e Medicina de Emergência (2). Todos relataram ter realizado ou assistido a drenagem torácica em pacientes in vivo, em média por 100 vezes. Os profissionais responderam a um questionário de percepção sobre o manequim, cujos dados estão presentes na Tabela 3.

Tabela 3. Resultados do questionário de percepção aplicado aos profissionais sobre o modelo de drenagem torácica

\begin{tabular}{|c|c|c|c|c|c|}
\hline Perguntas & CT & $\mathbf{C P}$ & $\mathbf{I N}$ & DP & DT \\
\hline O modelo pode ser usado para ensino de alunos da graduação. & $100 \%$ & $0 \%$ & $0 \%$ & $0 \%$ & $0 \%$ \\
\hline $\begin{array}{l}\text { Antes de realizar o procedimento em pacientes, o treino em um } \\
\text { manequim como esse deve ser realizado. }\end{array}$ & $100 \%$ & $0 \%$ & $0 \%$ & $0 \%$ & $0 \%$ \\
\hline O modelo é realístico. & $50 \%$ & $50 \%$ & $0 \%$ & $0 \%$ & $0 \%$ \\
\hline O modelo é reprodutível por outros centros de ensino. & $100 \%$ & $0 \%$ & $0 \%$ & $0 \%$ & $0 \%$ \\
\hline
\end{tabular}

Legenda: CT: concordo totalmente; CP: concordo parcialmente; IN: indiferente; DP: discordo parcialmente; DT: discordo totalmente.

Diante da versão final do modelo, os autores puderam usá-lo em edições do curso teórico-prático de procedimentos invasivos. A amostra foi composta por um total de 129 estudantes de graduação em Medicina que realizaram individualmente o procedimento, sendo $41,9 \%$ do sexo masculino e $58,1 \%$ do sexo feminino, com idades variáveis (entre 17 e 32 anos) e pertencentes a 7 instituições de ensino distintas, tanto do estado do Ceará quanto do Rio

Tabela 4. Sequência de passos durante o procedimento de drenagem torácica $(n=129)$

\begin{tabular}{lccc}
\hline Checklist & Acerto & Erro & $p$ \\
\hline Seleção do material & $65,1 \%$ & $34,9 \%$ & $<0,05$ \\
Posicionamento do paciente & $67,4 \%$ & $32,6 \%$ & $<0,05$ \\
Local da incisão & $76,0 \%$ & $24,0 \%$ & $<0,05$ \\
Paramentação cirúrgica & $88,4 \%$ & $11,6 \%$ & $<0,05$ \\
Assepsia e antissepsia & $85,3 \%$ & $14,7 \%$ & $<0,05$ \\
Anestesia & $58,9 \%$ & $41,1 \%$ & $<0,05$ \\
Incisão da pele & $76,0 \%$ & $24,0 \%$ & $<0,05$ \\
Divulsão & $74,4 \%$ & $25,6 \%$ & $<0,05$ \\
Exploração digital & $82,2 \%$ & $17,8 \%$ & $<0,05$ \\
Medição do dreno & $89,1 \%$ & $10,9 \%$ & $<0,05$ \\
Inserção correta do dreno & $64,3 \%$ & $25,7 \%$ & $<0,05$ \\
Conexão ao sistema de drenagem & $79,1 \%$ & $20,9 \%$ & $<0,05$ \\
Fixação do tubo & $83,7 \%$ & $16,3 \%$ & $<0,05$ \\
\hline
\end{tabular}

Grande do Norte. Destes, 93,7\% estavam no ciclo básico do curso de Medicina e 95,3\% não apresentavam nenhuma experiência com o procedimento de drenagem torácica (teórica ou prática). O resultado encontrado na correção do checklist, bem como acertos e erros na realização da drenagem torácica no simulador proposto estão resumidos nabela 4. 


\section{DISCUSSÃO}

Sabe-se que, na educação médica, os estudantes percorrem níveis para aprofundar o conhecimento sobre competências adquiridas no treino de procedimentos ${ }^{5,13}$. Um desses níveis contempla a habilidade de demonstrar a técnica proposta em ambiente de simulação, o que foi proposto durante a aplicação do modelo de drenagem torácica no curso teórico-prático.

Foi visto que este simulador dispõe de uma grande diferença de preço quando comparado a modelos comerciais, que custam em torno de R $\$ 10.000,00 /$ US\$ 2.500. Levando-se em consideração que a maioria das simulações com procedimentos invasivos no âmbito acadêmico são realizados com manequins comerciais de alto custo, este modelo pode ser uma alternativa acessível devido ao uso de materiais sintéticos de baixo custo, tendo uma reprodutibilidade maior ${ }^{6,14}$.

No que concerne a avaliação do simulador de drenagem torácica por médicos especialistas todos concordaram totalmente quanto ao seu uso no ensino da técnica para estudantes de Medicina. Também concordaram que apresenta parâmetros anatômicos e funcionais que viabilizam sua utilização pelos acadêmicos como equipamento de treino antes de realizar o procedimento em pacientes reais ${ }^{13,15}$.

Quanto à percepção sobre a correlação anatômica, verificou-se que o modelo apresenta limitação quanto a sensação tátil da pleura durante execução da exploração digital, que visa localizar corretamente a inserção do dreno. Porém, mesmo com tal limitação, o modelo permite o treino de todos os passos da técnica da drenagem torácica de forma satisfatória.

Com relação aos passos do checklist, as etapas com maiores índices de acertos pelos alunos foram a assepsia e antissepsia, paramentação adequada e identificação do local apropriado para incisão. Estes pontos iniciais básicos são de suma importância, visto que, quando realizados corretamente, geram menos riscos de falhas no procedimento e de complicações ao paciente ${ }^{16}$.

Entretanto, maiores percentuais de erro são vistos nos itens: seleção adequada do material, posicionamento correto do paciente e aplicação da anestesia. As falhas nestes itens podem ser explicadas pela falta do contato prévio com o procedimento, visto que a extensa maioria dos acadêmicos não apresentava experiência teórica ou prática prévia. Apesar do exposto, pode-se perceber que mesmo sem um contato prévio com o procedimento, a maioria dos acadêmicos conseguiu executar de forma suficiente a técnica proposta.

Observou-se também que o treino baseado no checklist permite simples checagens antes e durante do procedimento, contribuindo para impedir complicações e, consequentemente, para a redução da morbimortalidade dos pacientes ${ }^{17}$.

O uso de simuladores de baixo custo como este permite que o aluno treine em diferentes cenários, minimizando a possibilidade de futuras intercorrências no procedimento por falha de técnica. Além disso, essa metodologia possibilita o feedback em tempo real, gerando um desenvolvimento educacional que permite o aprendizado a partir dos erros, aprimorando a técnica ${ }^{18,19}$. Isso reforça a relevância de propagar o uso de simuladores acessíveis para o uso em outras escolas médicas.

\section{CONCLUSÕES}

Tendo em vista o baixo custo e o fácil acesso aos materiais sintéticos utilizados, percebe-se que o simulador de drenagem de tórax descrito apresenta reprodução possível nos centros universitários, tornando-o mais uma ferramenta disponível para a capacitação de alunos da graduação. Há ainda a necessidade de ampliar os estudos com modelos de procedimentos invasivos de baixo custo, para que possam ser usados em larga escala no ensino médico.

Participação dos autores: Oliveira MA: Montagem do modelo, coleta de dados, redação do artigo, elaboração de figuras e revisão final. Queiroz EF: Montagem do modelo, análise de dados, redação do artigo, elaboração de tabelas e revisão final. Mesquita DAK: Revisão de literatura, coleta de dados e redação do artigo. Marques LM: Revisão de literatura, tabulação de dados e redação do artigo. FM: Orientação do projeto e revisão final. Correa $R V$ : Orientação do projeto, redação do artigo e revisão final.

\section{REFERÊNCIAS}

1. Marques RG. Técnica operatória e cirúrgica experimental. Rio de Janeiro: Guanabara Koogan; 2005.

2. Ciprono FG, Dessote LU. Drenagem pleural. Medicina (Ribeirão Preto). 2011;44(1):70-8. https://doi.org/10.11606/ issn.2176-7262.v44i1p70-78.

3. Brasil. Conselho Nacional de Educação. Câmara de Educação Superior. Resolução CNE/CES No 4, de 7 de novembro de 2001. Institui Diretrizes Curriculares Nacionais do Curso de Graduação em Medicina [resolução na internet]. Diário Oficial da União, 7 nov. 2001 [citado 14 fev. 2019]. Disponível em: http://portal.mec.gov.br/cne/arquivos/pdf/CES04.pdf.
4. Flato UA, Guimarães HP. Educação baseada em simulação em medicina de urgência e emergência: a arte imita a vida. Rev Bras Clin Med. 2011;9(5):360-4. Disponível em: https://pdfs.semanticscholar.org/fb32/ f4811a8a6e84912e4483ed3a00d756acb329.pdf.

5. Lateef F. Simulation-based learning: Just like the real thing. J Emerg Trauma Shock. 2010;3(4):348-52. https://doi. org/10.4103/0974-2700.70743

6. Bettega AL, Brunello LF, Nazar GA, De-Luca GY, Sarquis LM, Wiederkehr HA et al. Simulador de dreno de tórax: desenvolvimento de modelo de baixo custo para 
capacitação de médicos e estudantes de medicina. Rev Col Bras Cir. 2019;46(1):e2011. https://doi.org/10.1590/01006991e-20192011

7. Tube MI, Netto FA, Costa E, Lafayette DS, Lima GA, Menezes JI, et al. Chest drainage teaching and training for medical students. Use of a surgical ex vivo pig model. Acta Cir Bras. 2016;31(5):353-63. https://doi.org/10.1590/S0102865020160050000010

8. Netto FA, Sommer CG, Constantino MM, Cardoso M, Cipriani RF, Pereira RA. Projeto de ensino: modelo suíno de baixo custo para treinamento de drenagem torácica. Rev Col Bras Cir. 2016;43(1):60-3. https://doi.org/10.1590/010069912016001012 .

9. Carter YM, Brette BM, Hall E, Marshall MB. Multipurpose Simulator for technical skill development in thoracic surgery. J Sur Res. 2010;163(2):186-91. https://doi.org/10.1016/j. jss.2010.04.051.

10. Shelly PD, Bartolomeu Junior N, Carmine S, Vincent C. Chest-Tube insertion. N Engl J Med. 2007. 357:e15. _https:// doi.org/10.1056/NEJMvem071974.

11. Havelock T, Teoh R, Laws D, Gleeson F; BTS Pleural Disease Guideline Group. Pleural procedures and thoracic ultrasound: British Thoracic Society pleural disease guideline. Thorax. 2010;65(Suppl 2):61-76. https://doi.org/10.1136/ thx.2010.137026.

12. Zamboni V. Suporte avançado de vida no trauma. In: Scalabrini NA, Dias RD, Velasco IT. Procedimentos em emergência. São Paulo: Manole; 2016. p.41-43.

13. Pereira CM, Vita PM. A simulação como metodologia de aquisição de competências na formação médica pré-graduada
[Dissertação]. Porto: Universidade do Porto; 2011. Disponível em: https://repositorio-aberto.up.pt/bitstream/10216/62207/2/ Ctia\%20Pereira\%20Dissertao.pdf.

14. Maluf Junior I, Silva AB, Groth AK, Lopes MA, Kurogi AS, Freitas RS, et al. An alternative experimental model for training in microsurgery. Rev Col Bras Cir. 2014;41(1):72-4. https://doi.org/10.1590/S0100-69912014000100014.

15. Issenberg SB, Scalese RJ. Five Tips for a Successful Submission on Simulation-Based Medical Education. J Grad Med Educ. 2014;6(4):623-5. https://doi.org/10.4300/ JGME-D-14-00490.1.

16. Mendes CA, Hirano ES. Predictors of chest drainage complications in trauma patients. Rev Col Bras Cir. 2018;45(2):e1543. https://doi.org/10.1590/01006991e-20181543.

17. Pancieri AP, Carvalho R, Braga EM. Aplicação do checklist para cirurgia segura: relato de experiência. Rev Sobecc. 2014;19(1):26-33. https://doi.org//10.4322/sobecc.2014.006.

18. Marcieira LMM, Teixeira MCB, Saraiva JMA. Simulação médica no ensino universitário de pediatria. Rev Bras Edu Med, 2017;41(1):86-91. https://doi.org/10.1590/1981$52712015 \mathrm{v} 41 \mathrm{n} 1 \mathrm{rb} 20160032$.

19. Dourado ASS, Giannella TR. Ensino baseado em simulação na formação continuada de médicos: análise das percepções de alunos e professores de um hospital do Rio de Janeiro. Rev Bras Edu Med. 2014;38(4):460-469. https://doi.org/10.1590/ S0100-55022014000400007.

Recebido: 05/08/2019

Aceito: 04/03/2020 